\title{
Quantum Mechanical Aspect in Bayesian Optimization
}

\author{
Ichio Kikuchi and Akihito Kikuchi*
}

December 25, 2019

\begin{abstract}
In this essay, we point out the quantum mechanical aspect in Bayesian Optimization: one might interpret Bayesian optimization as a process of computation in quantum mechanics, especially that of the molecular orbital method. We demonstrate that the optimization of the molecular structure and the refinement of the basis set is the process of Bayesian optimization, in which one should try to find better sampling points. The Bayesian optimization based upon the Gaussian process would be substituted by the molecular orbital computation using atomic orbitals of Gaussian functions. Besides, we propose a trick which would act as a sort of acquisition function. We give some examples in the polynomial optimization.
\end{abstract}

\section{Preliminaries}

Nowadays Bayesian optimization is in fashion in almost all of the field of scientific and industrial computations where one would encounter with the optimization of the objective functions: namely, to find the maximum of the function:

$$
\max _{x} f(x) .
$$

There are various methods to solve this question: usually they adopt the set of sampling points in the topological space, compute the values of the objective functions, and find the optimum through the comparisons. As one could not see through the whole space, the numbers of the samples are limited; thus one should choose them adroitly

\footnotetext{
*A.K. is the corresponding author: akihito_kikuchi@gakushikai.jp
} 
in the restricted resource. Bayesian Optimization is one of the effective methods of this kind $[1,2]$. It is based on the likelihood problem (or the probability) and it would not demand us the computation of the derivatives of the objective function. (If one can compute the derivative, it would suggest the new direction in which one should take the new sampling. The extra computation of the derivatives would, however, cause more computational costs; if the function is not given by the analytic form, the exact computation of derivatives is impossible.)

The process of Bayesian optimization would be summarized as follows. Let us denote $x_{i}$ as the i-th sample and the $f\left(x_{i}\right)$ is the observation of the objective function. The iterated observation are stored in $\mathbf{D}_{1: t}=\mathbf{x}_{1: t}, \mathbf{f}\left(x_{1: t}\right)$. (Here the notation $\mathbf{x}_{1: t}$ signifies the sequence $x_{1}, x_{2}, . . x_{t}$.)

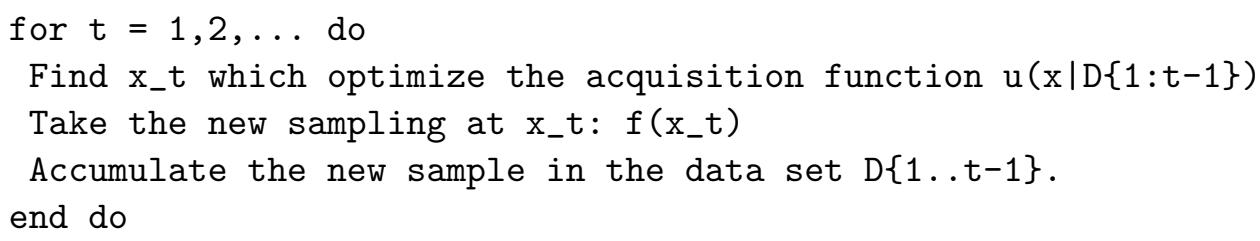

The new sample point is chosen in such a way that the acquisition function $u\left(x \mid D_{1: t-1}\right)$ would take the maximum at this point, so that one could refrain oneself from "feeling for" the optimum haphazardly. The acquisition function is composed of past sampling and one could guess the sampling point which would improve the optimization.

In Bayesian optimization, several variants of acquisition function have been proposed. For example, one should minimize the deviation of the current optimum from the true one: the expectation should be minimized.

$$
x_{t+1}=\operatorname{argmin}_{x} E_{n}\left[f(x)-f^{*} \mid D_{1 . . t-1}\right]
$$

The practical computation of this principle would be done by

$$
x_{t+1}=\operatorname{argmin}_{x} E_{n}\left[\max \left\{0, E\left(X^{+}\right)-H\right\} \mid D_{1 . . t-1}\right] .
$$

Or one can choose this scheme: the increase (or the gain) of the next posterior distribution $\mu_{n+1}$ from the current $\mu_{n+1}$ should be maximized in each step of the new sampling point choice. It is called the knowledge gradient and the following function (of the expected increase) should be maximized:

$$
K G_{n}(x)=E_{n}\left[\mu_{n+1}-\mu_{n} \mid x_{n+1}=x\right] .
$$




\section{Formalism with the view of quan- tum mechanics}

Let us review the computational process of the optimization from the standpoint of quantum mechanics $[3,4]$. One should interpret the sample point $x_{i}$ as the quantum state $\left.\mid x_{i}\right)$. This state, as a wave-function, should be centered at $x_{i}$, and its square represents the probability distribution; but it would not be a point: rather it would be a distribution around the center; in the limit, the distribution would be a delta-function and the spatial integration $\int x \delta(x-a) d x$ would give back the position of the center.

The objective function $V(x)$ would be represented by the Hamiltonian operator:

$$
H=-\frac{h^{2}}{2} \nabla+V(x),
$$

where the small kinetic term is added.

The sampling is to compute the expectation value $\left(x_{i}|H| x_{i}\right)$. Concerning this, we should execute the minimization, because we apply the energetic of quantum mechanics where the physical properties are bounded from below.

As Bayesianists, let us stand at the situation where (t-1) samplings have been done. We have ( $\mathrm{t}-1)$ states $\left.\mid x_{i}\right)$ and $(\mathrm{t}-1) \times(\mathrm{t}-1)$ matrix elements $\left(x_{i}|H| x_{i}\right)$. How could we choose the new sampling point? Now we should consider the problem in quantum mechanics. At least we could find the eigenvalues of the operator $H$ using the finite sequence of the basis set $\left(x_{i}\right)$. The lowest eigenvalue would be the current best in the optimization because it is the center of the wave-function (that is to say, the center of the probability distribution). We should refine the basis set by introducing the new $\left.\mid x_{t}\right)$ around the point $x_{t}$ : we should place it at the point in which the objective function would decrease (or would be improved). Or we should alter the position of atoms totally so that the energy should take the minimum. The requirement is how to construct the acquisition function.

We can employ the analytic basis set. In the atomic physics, the popular one is the Gaussian function (multiplied by polynomials). Let us simply define it by

$$
\left(r \mid x_{i}\right)=C \exp \left(-\left(r-x_{i}\right)^{2}\right) .
$$

The constant $C$ is placed so that $C^{2} \int \exp \left(-2\left(r-x_{i}\right)^{2}\right) d r=1$.

The optimum is the lowest eigenvalue of the secular equation. As the chosen basis set is not ortho-normalized, one should solve the generalized eigenvalue problem in the following form:

$$
(H-e K) X=0,
$$


where the Fock matrix $H$ and the overlapping matrix $K$ are defined by

$$
\begin{aligned}
H_{i, j} & =\left(x_{i}\left|-\frac{h^{2}}{2} \nabla+V(r)\right| x_{j}\right), \\
K_{i, j} & =\exp \left(-1 / 2\left(x_{i}-x_{j}\right)^{2}\right),
\end{aligned}
$$

and $e$ is the eigenvalue. The wave-function is represented by the entries of the eigenvector $X$ by

$$
\mid \psi)=\sum_{i=1}^{t-1}\left(x_{i}\right) X_{i} .
$$

The moment of the positional operators $x^{n}$ is evaluated by

$$
\left(\psi\left|x^{n}\right| \psi\right)
$$

For $\mathrm{n}=2$, the relation is always satisfied:

$$
\left(\psi\left|x^{2}\right| \psi\right)-(\psi|x| \psi)^{2} \geq 0,
$$

and the famous uncertainty relation between the kinetic moment and the position is also valid.

The surrogate function for $H$ is defined as

$$
f(x)=\sum_{i, j}\left(\phi_{x} \mid x_{i}\right)\left(K^{-1}\right)_{i, j}\left(x_{j}|H| \phi_{x}\right),
$$

where $\phi_{x}$ is the Gaussian $C \exp \left(-(r-x)^{2}\right)$. This function strictly returns the observed value $\left(x_{i}|H| x_{i}\right)$ at the point $x_{i}$, but it would be an approximation otherwise. It would give the expectation value of $H$ which a Gaussian placed at $x$ would read out.

The $\phi_{x}$ would belong to the basis set $\left.\mid x_{i}\right)$, according to the probability:

$$
P=\sum_{i, j}\left(\phi_{x} \mid x_{i}\right)\left(K^{-1}\right)_{i, j}\left(x_{j} \mid \phi_{x}\right)
$$

Now one shall see the analogy between above formulas of quantum mechanics and several fundamental formulas in Bayesian optimization: let us assume the Gaussian process; if we have the set of observations $D=\left\{\left(x_{i}, f_{i}\right)\right\}_{i=1}^{n}$, the probability, by which the observation at the point $x$ would fetch the value $y$, is given by:

$$
P\left(y \mid \mathbf{x}_{1: n}, \mathbf{f}_{1: n}\right) \approx \operatorname{Normal}(\mu(x), \sigma(x)) .
$$

In the above, the mean and the variance are defined from the covariance kernel $K$ :

$$
\mu(x)=\mathbf{K}\left(x, x_{1: n}\right) \mathbf{K}^{-1}\left(x_{1: n}, x_{1: n}\right) \mathbf{f}_{1: i},
$$


and

$$
\sigma^{2}(x)=\mathbf{K}(x, x)-\mathbf{K}\left(x, x_{1: n}\right) \mathbf{K}^{-1}\left(x_{1: n}, x_{1: n}\right) \mathbf{K}\left(x_{1: i}, x\right) .
$$

One should read the broad symbols $\left(\mathbf{K}(x, x), \mathbf{K}\left(x, x_{1: n}\right)\right.$ and $\left.\mathbf{K}^{-1}\left(x_{1: n}, x_{1: n}\right)\right)$ as the scalar, the vector and the matrix, respectively; the vector and the matrix are indexed from $x_{1}$ to $x_{n}$, and the indexed array is denoted by $x_{1: n}$.

In order to see the analogy, one should simply do the replacement as follows.

$$
\begin{gathered}
\mathbf{K}(x, x) \leftrightarrow\left(\phi_{x} \mid \phi_{x}\right)=1 \\
\mathbf{K}\left(x, x_{1: n}\right) \leftrightarrow\left(\phi_{x} \mid x_{i}\right) \\
\mathbf{K}\left(x_{1: n}, x_{1: n}\right) \leftrightarrow\left(x_{i} \mid x_{j}\right) \\
f_{i} \leftrightarrow\left(x_{i}|H| x_{i}\right)
\end{gathered}
$$

Through this substitution, the formulas of quantum mechanics (eq.(13) and eq.(14)) are the counterparts of the formulas in Bayesian optimization (eq.(16) and eq.(17)) Besides, if the states $\left.\mid \phi_{x}\right)$ is included in the space spanned by the basis set $\left.\left\{\mid x_{i}\right)\right\}$, the corresponding $\sigma^{2}(x)$ is zero: the observation is done without the ambiguity, in the existing basis set.

As is usual with the theme of Bayesian optimization, one would question these problems concerning our quantum mechanical model.

If one adds one more basis function in the computation, the lowest eigenvalue could decrease; there should be the best place for this basis function. What would it be? This is the question concerning the choice of the acquisition function.

The surrogate function should recover the objective function: what is the best arrangement of the basis set?

The plausible answer is extracted from the ground state of the wave function (or the quantum state with the lowest energy). And one should do the best choice of the basis set to execute the best computation. Now let us imagine the model of the one-dimensional well of the infinite depth. The probability distribution of the ground state will be centered at the center of the well; on the other hand, the second-lowest has two peaks of the distribution; it would be approximated by two Gaussian functions. If one puts another Gaussian at the center of the well, the result of the computation would be improved because the right and the left Gaussian function would be eclipsed by the center one in the weight in the ground state of the wave-function. 


\section{More analogy to molecular orbital theory and numerical experiment}

In the previous section, we set up the optimization using the eigenvalue problem, where the parameters of the variation would the positions of the centers of the Gaussian-type orbitals: the optimum would be given by the minimum of the lowest eigenvalue. This is the well-known situation of the optimization of the molecular geometry, where the atomic orbitals are represented by the Gaussian-type orbitals which are located at atoms; and the wave-function would be the linear combination of the atomic orbitals. Through this analogy, we would be able to find the minimum of some function $W(x)$ by using the imaginary molecule as a probe. The molecule would be placed in the potential function $W(x)$ and it would be located around the bottom of the function when the molecule would take the stablest configuration.

The actual molecule is stabilized by the balance of repulsive and attractive forces: in quantum theory, they are represented by the electrostatic interaction and the more complicated quantum effects among the nuclei and the electrons. Although the interaction among electrons is non-linear concerning the wave-function, the simple eigenvalue problem, as is given in the previous section, does not include such nonlinear terms and it would fail to be a quantitative description of the quantum dynamics. Thus in the simplified style of molecular orbital computation (which neglects the non-linear quantum mechanical effect), one would often approximate the total energy of the molecule by the two terms: the one is the summation of the eigenvalues and the another is an extra empirical repulsive potential energy function concerning the atomic coordinates $[8,9]$. Using this approximation, we might customize our objective function by the sum of the lowest eigenvalue and a repulsive potential function:

$$
f(\phi)=(\phi|H| \phi)=\left(\phi\left|-h_{q} \nabla^{2}+W\right| \phi\right)+\frac{1}{N(N-1)} \sum_{i>j} V_{r e p}\left(x_{i}, x_{j}\right),
$$

where $(\phi|\ldots| \phi)$ is the lowest eigenvalue of the eigenvalue problem given in the previous section. We use $\mathrm{N}$ orbitals, centered at $x_{i}$, to construct the sampler molecule. Here $h_{q}$ is the constant, upon which the quantum dynamical feature would depend. (We omit the denominator 2 in the quantum mechanics for simplicity.)

As for the repulsive empirical potential, we adopt the LennardJones potential:

$$
V_{\text {rep }}\left(x_{i}, x_{j}\right)=V\left[-\left(\frac{r_{0}}{\left|x_{i}-x_{j}\right|}\right)^{6}+\left(\frac{r_{0}}{\left|x_{i}-x_{j}\right|}\right)^{12}\right] .
$$


This function is weakly attractive in the long range and sharply repulsive in the short range. It would depict the hard-core character of a particle of finite volume.

As for the object function (e.g. the electronic potential) $W(x)$ we adopt an arbitrary polynomial:

$$
W(x)=C_{0}+C_{1} x+C_{2} x^{2}+\ldots+C_{N} x^{N} .
$$

The polynomial can be multivariate.

The atomic orbital is defined by the general style:

$$
\left(r \mid x_{i}\right)=\left(\frac{\pi}{2 a}\right)^{1 / 4} \exp \left(-a x^{2}\right)
$$

so that the radius of the atomic orbital would be altered. ${ }^{1}$ In the molecular orbital computation, the gradient of the energy can be represented by the rigorous analytic form, from which we can execute numerical computation. However, for simplicity, we use here expedient ways (which enables us to do without "derivatives", such as particle swarm optimization or the genetic algorithm. These algorithms, in general, cultivate a group of the samplers and propagate them toward the optimum, so that the practitioner would finally elicit the best one from the group.

According to this condition, a simple computation is done in the one-dimensional model. The figures (Fig.1 and 2) show the result of the computation for obtaining the optimum surrogate function around the bottom of the potential function. The adopted algorithm for seeking the lowest energy is particle swarm optimization. The surrogate function is computed by the expectation value of the polynomial potential $W(x)$.

The test objective function is the polynomial of a fourth-degree, the shape of which is slightly asymmetric. The initial probability distribution shrank into the optimum one. The final surrogate function depicts the inclined curve of the objective function. The similarity between the computed surrogate function and the exact object function would be improved by adjusting the width of the Gaussian orbital.

\footnotetext{
${ }^{1}$ The integrals involved in the eigenvalue problem are computed exactly. One might utilize the formula: $\int_{\infty}^{\infty} \exp \left(-a x^{2}+b x+c\right)=\exp \left(\frac{b^{2}}{4 a^{2}}+c\right)$. The derivatives with respect to $a$ and $b$ shall give us the integrals of the Gaussian multiplied by the powers of $x$.
} 


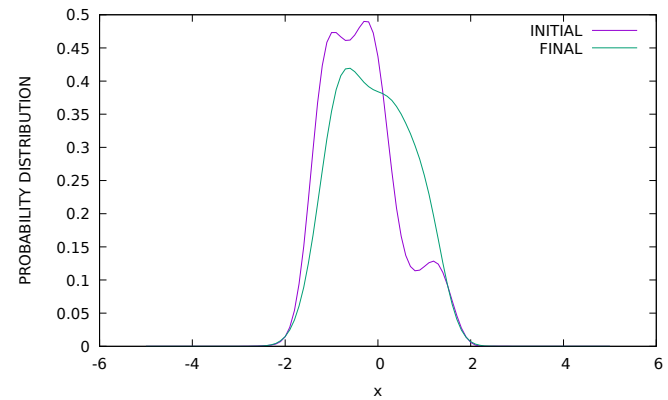

A

Figure 1: The probability distribution of one of the sample molecule (initial / final) by the optimization on the polynomial object function $x^{4}-2 x^{2}+0.1 x$. The parameters are chosen as follows: as for quantum mechanical part, the Gaussian width is $a=2$ and the coefficient of the kinetic energy is $h^{2}=1$; for the classical part, the magnitude of the repulsive term is $V=1 / 2$ and the effective range is $r_{0}=0.5$. The optimization is done by the particle swarm optimization, by means of 10 samplers.

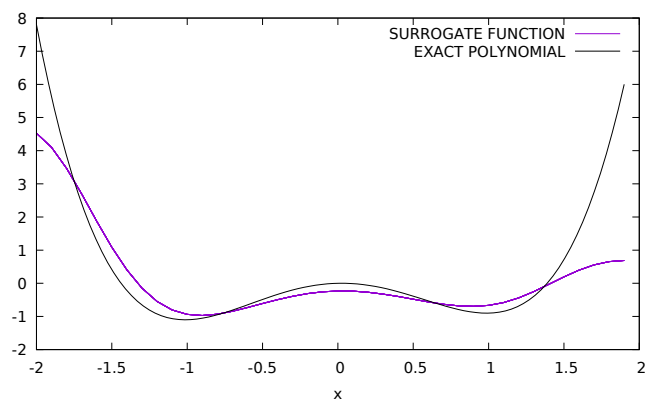

Figure 2: The computed surrogate function and the exact object function, from the computation in Fig.1 
2D example. Gaussian Contraction Coefficient $=2$

Surrogate function Exact function

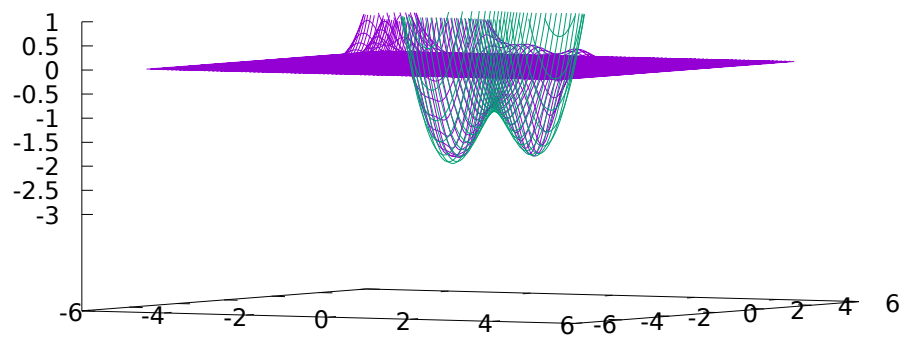

2D example. Gaussian Contraction Coefficient $=8$

Surrogate function

Exact function

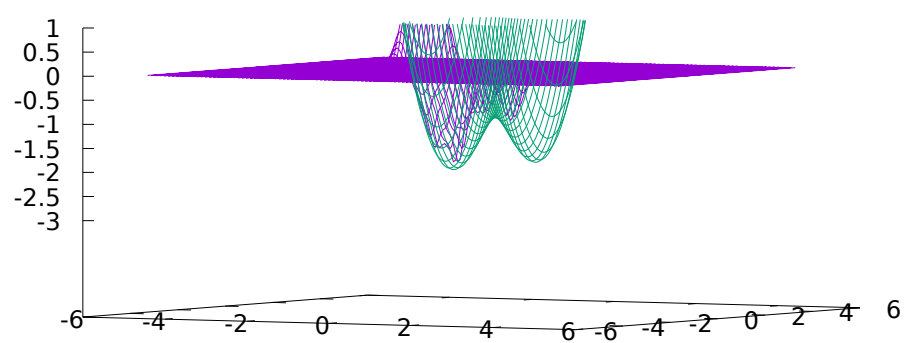

Figure 3: The computed surrogate function and the exact object function by a two-dimensional computation. The upper and the lower figures shows the results with different Gaussian contraction coefficient $\left(a\right.$ at $\left.\exp \left(-a x^{2}\right)\right), 2.0$ and 8.0. 
Fig. 3 shows the influence of the Gaussian width on the depicted surrogate surfaces. This computation is done for a two-dimensional case, where the potential function is $x^{4}-2 x^{2}+0.1 x+y^{2}-2 y 2 .$. The upper figures use the broader Gaussians than the lower figure does. The broader Gaussians reproduce the two valleys of the potential function. The narrower Gaussians also detect the valleys, but owing to the interaction among them, the orbital in one valley is attracted to the other orbitals in another valley, and it could not touch the bottom. The computed surrogate function is almost zero in the regions where the Gaussians are nearly zero. The rises in the computed results are located at the position of the Gaussians which scarcely contribute to the quantum ground state: they remain outside the optimum wavefunction because they are set apart during the optimization. To obtain the better result, we should employ these tactics, as are common in Bayesian optimization: (1) one should also optimize the Gaussian width; (2) one should add extra Orbitals, to fill hollows of the less reliable parts of the surrogate function. Even if some Gaussians are not be bound to the Ground state and remain outside, they would contribute to the higher quantum state (which would extend more broadly in space). The optimizations for the higher quantum state would be effective to fully utilize the Gaussian basis set.

\section{Discussion}

Now we propose a trick by which one could do the effective computation. As aforementioned, the center of the probability distribution would suggest the best point at which the atomic orbital should be placed. One might place an extra orbital at this point; one might shift the whole of the molecule so that one of the existing atomic orbitals would be placed here. The latter approach might be more preferable in some cases because the cost of solving the eigenvalue problem would be dominating when the size is large. One should avoid the growth of size by keeping it constant.

We adopt this principle.

If the computed lowest eigenvalue of a sampler molecule is lower than any of the object function observed at the atoms $\left(x_{i}|H| x_{i}\right)$ (which are the orbital energies on the diagonals of the Fock matrix), the atoms are moved in parallel so that the center of the sampler molecules is shifted to the center of the probability distribution.

As the relative position of the molecule concerning the external potential (the objective function) would vary, this treatment could 
not be useless. The efficacy of this trick is shown in the computation in Fig.4, where the comparison is done with the two cases with or without the trick. The results suggest that the proposed treatment would be effective, to avoid the local minima, which trapped the gait of the computation without the trick.

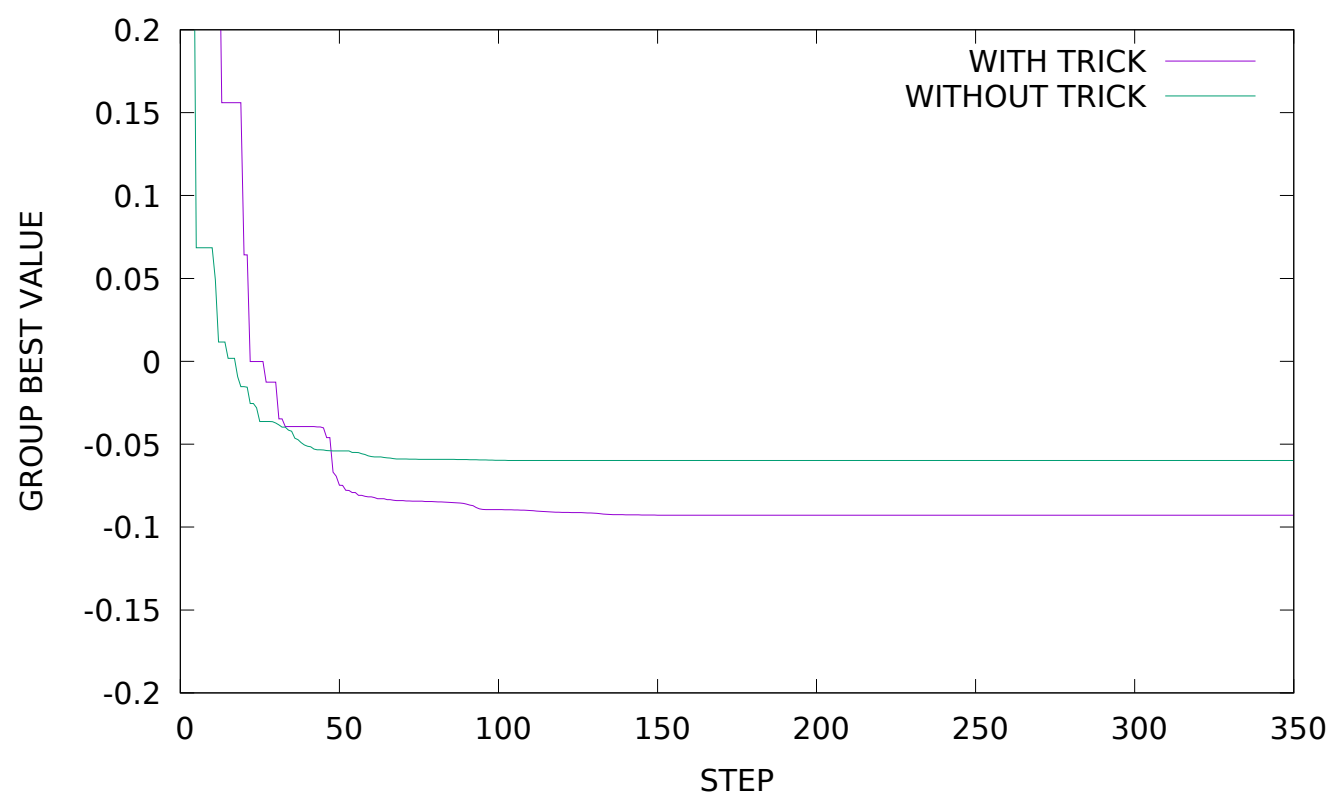

Figure 4: The improvement by the trick explained in the article. The computation with that trick avoids being trapped into a local minimum, which has caught the computation (without the trick). The computation is done with the same setting as in Fig.1

As for the surrogate function, one might use the inversion formula as follows:

$$
V(x)=E+\frac{d^{2} \phi(x) / d x^{2}}{\phi(x)} .
$$

The evaluation of effective potential is successful in the atomic problem, concerning the pseudo-potential generation when the wavefunction is defined on the mesh points. However, in our method, the Gaussian basis set is too coarse to reproduce the potential by this formula, if it is not well chosen. For example, let us consider the model with the test function $\phi(x)=C \exp \left(-a x^{2}\right)$ and $H=-d^{2} / d x^{2}+x^{4}$. Then $V(x)=(\phi|H| \phi)-2 a+4 a^{2} x^{2}$. The trial function cannot reproduce the arbitrary potential, even if the function exactly points out the optimum. In the quantitative computation of quantum chemistry, 
one must adopt the extended basis set which is represented by the Gaussian and the polynomial.

There is another left question. In the exemplary computations, we adopt the objective function of the polynomial. We do the "observation" by computing the analytic formula. In general case, however, the analytic formula would not be obtainable, and we use the "observed values" apart from the optimization program. In our construction, the diagonal terms in the Hamiltonian would be supplied with these values. As for the off-diagonals, we need some approximation. In the empirical molecular orbital theory, one often adopts the extended Hückel approximation in these sense $[5,6,7]$ :

$$
H_{i j}=K \frac{H_{i i}+H_{j j}}{2} \cdot S_{i j}
$$

where $\mathrm{K}$ is an adjustable constant so that one would approximate the off-diagonal terms by averaging the diagonal terms. In fact, in our computational process, this approximation is usable as it is in the diagonalization. The surrogate function might be treated with care: it is defined as

$$
\left(\phi_{x}|H| \phi_{x}\right)=\sum_{i, j}\left(\phi_{x} \mid x_{i}\right)(K)_{i, j}^{-1}\left(x_{j}|H| \phi_{x}\right) .
$$

The right side would include the value of the surrogate function itself. So it might be approximated by

$$
\left(\phi_{x}|H| \phi_{x}\right)=\left(\phi_{x} \mid x_{i}\right)(K)_{i, j}^{-1}\left(x_{j}|H| x_{j}\right)\left(x_{j} \mid \phi_{x}\right) .
$$

Or it might be computed from a linear equation:

$$
\left(\phi_{x}|H| \phi_{x}\right)=\sum_{i, j}\left(\phi_{x} \mid x_{i}\right)(K)_{i, j}^{-1}\left(\left(x_{j}|H| x_{j}\right)+\left(\phi_{x}|H| \phi_{x}\right)\right) / 2 .
$$

\section{Concluding Remark}

In this article, we construct the Bayesian optimization with the flavor of quantum dynamics. The assumption of the computation would be summarized as these statements. (1)The principle objective function $W(x)$ is a polynomial. (2)One sampler of the optimization is a "molecule": it is composed of N-atomic orbitals: one atomic orbital is placed on one atom. The atoms should be set apart and intervened by the modest distances. (3)The repulsive interaction is placed among the atoms so that the molecule would not collapse. (4)The molecular geometry should be optimized in the potential of $W$ so that the molecule would lie around the bottom of the potential. The 
simple example, computed there, would suggest the power of quantum simulation as the tool to solve the optimization problem in the more general context. The optimization would be done through the empirical molecular orbital theory, merely by computing the lowest eigenvalue of the Hamiltonian. No doubt the present simple model should be improved: the computation involve the solution of the generalized eigenvalue problem, which would be expensive in the large size of the computation. Concerning this, some order- $\mathrm{N}$ method (the computational cost of which is proportional to the matrix size) have been proposed, and they would be useful[10, 11]. The present model is basically the one-electron ground-state problem. But we can treat the multi-electron case. For example, we might treat the excited state, where the electron lies in the higher energy state. In the excited state, the electronic wave would expand more broadly, and it would cover the different bottom of the potential; if we sooth down the excited state to the ground state, it would choose the optimum, which would be hidden from other local minima. One could take into account the interaction between several electrons. The computed quantum states would occupy the local minima from the lowest to the highest upwardly: the electrons localized at one minimum and another minimum would be spatially exclusive because of the electrostatic interaction. If one could make a proper but easily computable model of the electrostatic repulsion, such as the Hubbard model, this would bring us an orderly computational process of finding out the local minima. (The use of the long-ranged electrostatic potential would bring us the same problem which would baffle the practitioner of the molecular orbital method: it would incite the intractable many-body problem. Thus one must replace the electrostatic potential with a more simple style.) The authors believe that these unexploited themes would be the clues to the more competent algorithm and present them to the readers as open problems.

\section{References}

[1] Eric Broch, Vlad M. Cora and Nando de Fereitas, "A Tutorial on Bayesian Optimization of Expensive Cost functions, with Application to Active User Modeling and Hierarchical Reinforcement Learning", arXiv:1012.2599v1, 2010.

[2] Peter I. Frazier, "A Tutorial on Bayezian Optimization", arXiv:1807.02811v1, 2018.

[3] P.A.M. Dirac, "The Principles of Quantum Mechanics", FOURTH EDITION, Oxford University Press, 1958. 
[4] Attila Szabo, Neil S. Ostlund, "Quantum Chemistry: Introduction to Advanced Electronic Structure Theory", Dover Books on Chemistry,1996.

[5] R. Hoffmann, "An extended Hückel theory. I. hydrocarbons", The Journal of Chemical Physics, 39(6), p1397-1412, 1963.

[6] D. Kienle, J. I. Cerda, and A. W. Ghosh, "Extended Hückel theory for band structure, chemistry, and transport. I. Carbon nanotubes", Journal of applied physics,100(4), p043714,2006.

[7] D. Kienle, K.H. Bevan, G-C Liang, L. Siddiqui, J.I. Certa, and A. W. Ghosh, "Extended Hückel theory for band structure, chemistry, and transport. II. Silicon", Journal of applied physics, 100(4), p043715,2006.

[8] , D.J. Chadi, "Atomic and electronic structures of reconstructed Si (100) surfaces",Physical Review Letters, 43(1), p43,1979.

[9] , C.M. Goringem D.R. Bowker and E. Hernandez, "Tightbinding modeling of materials", Reports on Progress in Physics, 60(12),p1447,1997.

[10] C. S. Jayanthi, S. Y. Wu, S. Y., J. Cocks, N. S. Luo, Z. L. Xie, M. Menon, M., and G. Yang, "Order- $N$ method for a nonorthogonal tight-binding Hamiltonian", Phys. Rev. B, 57(7), p3799-3802,1998.

[11] P. Ordejón, "Order-N tight-binding methods for electronicstructure and molecular dynamics", Computational materials science, 12(3), p157-191,1998. 